\title{
Multilevel Compression Scheme using Vector Quantization for Image Compression
}

\author{
S.Vimala, B.Abidha, and P.Uma
}

\begin{abstract}
In this paper, we have proposed a multi level compression scheme, in which the initial codebook and the Index Map that are generated using the normal Vector Quantization are compressed in three levels. The Minimum Mean Square method that is adopted in Block Truncation Coding is used to compress the codebook in first level with some improvements. The interpolative method is used to perform second level of compression to the codebook. Finally the Search Order Coding method is used to compress the Index Map without loss of image data. The experimental results show that there is a significant improvement in bit reduction without loosing much of image data.
\end{abstract}

Index Terms-codebook, compression, index-map, interpolative, MMSE, SOC

\section{INTRODUCTION}

Recent researches concentrate in finding ways to code images efficiently using digital techniques for image processing [1]. Generally images require large amount of data resulting in consumption of huge bandwidth and storage resources. Image compression is essential for applications such as TV transmission, video conferencing, facsimile transmission of printed material, graphics images, or transmission of remote sensing images obtained from satellites and reconnaissance aircraft [2]. Vector Quantization (VQ) [3]-[5], due to its simplicity and coding efficiency, has been successfully used in various applications involving VQ based encoding and VQ based recognition. $\mathrm{VQ}$ is an efficient image coding technique achieving a bit rate, less than one bit per pixel (bpp). Different types of VQ, such as classified VQ [6], [7], address VQ [8], finite state VQ [9], Side match VQ [10], Mean-removed classified VQ [11] and Predictive classified VQ [9], [11] have been used for various purposes. VQ comprises of three stages: codebook designing, image encoding and image decoding.

A Vector Quantizer $\mathrm{Q}$ of dimension $\mathrm{k}$ and size $\mathrm{N}$ is a mapping from a vector in k-dimensional Euclidean space points [14]. This can be expressed mathematically as $Q: R^{k}$ $\rightarrow \mathrm{C}$, where $\mathrm{C}=\left\{Y_{1}, Y_{2}, Y_{3}, \ldots, Y_{M}\right\}$ and $Y i \in R^{k}$. The set $\mathrm{C}$ is called the codebook and $Y i, \quad l<=i<=M$ are called codewords.

The codebook generation is the important task in VQ. [12]

Manuscript received May 28, 2011; revised December 6, 2011.

The authors are with the Department of Computer Science, Mother Teresa Women's University, Kodaikanal - 624102, Tamil Nadu, India since 1999 (e-mail: vimalaharini@gmail.com, rabidha@yahoo.com, umapoomi@gmail.com).
Performance of the VQ is highly dependent on the optimality of the codebook. There are several known methods for generating a codebook [13]. LBG (Linde Buzo, Gray) [4] is the most widely referred VQ method for designing a Codebook.

The most widely used Generalized Lloyd Algorithm (GLA) starts with an initial solution, which is iteratively improved using two optimality criteria in turn until a local minimum is reached. It can also be built hierarchically. The iterative splitting algorithm [15], [16] starts with a codebook of size one, which is the centroid of the entire training set. The codebook is then iteratively enlarged by a splitting procedure until it reaches the desired size. Another hierarchical algorithm, the Pairwise Nearest Neighbor (PNN) [17], uses an opposite, bottom up approach to generate the codebook. The Vector Quantization algorithms for reducing the transmission bit rate or the storage have recently been extensively investigated for speech and image signals [18]. A fundamental goal of data compression is to reduce the bit rate for transmission or data storage while maintaining an acceptable fidelity or image quality.

In this paper, the compression rate is increased in multiple levels. The concepts of normal VQ with novel ideas incorporated, Minimum Mean Square Error (MMSE), Interpolative method and Search Order Coding (SOC) are integrated to reduce the bitrate to a greater extent. [19] The MMSE method and the Interpolative method [20] which are used in Block Truncation Coding (one other lossy image compression technique) are incorporated in VQ in the proposed scheme to reduce the bitrate to a significant extent. In the remaining part of the paper, the proposed scheme is described in Section 2, the results are discussed in Section 3 and the conclusion is given in Section 4.

\section{PROPOSED SCHEME}

In the proposed scheme, VQ is performed in different levels. A novel idea called the Codebook Generation with Edge Categorization (CBEC) is introduced to generate the initial codebook. Initially, the image is normal vector quantized with the novel idea incorporated in generating the codebook. The codebook thus generated during normal vector quantization is further compressed in three levels. In the first level, the codebook is compressed using Minimum Mean Square Error (MMSE). In the second level, the codebook is compressed using Interpolation method. In the third level, the concept of Search Order Coding (SOC) is used. The codebook thus generated and compressed is optimized using K-Means clustering in order to improve the quality of the codebook. The quality of the reconstructed image depends on the size of the codebook and the quality 
of the codebook. The methods involved in the proposed scheme are explained in the following sections.

\section{A. Codebook Generation with Edge Categorization (CBEC)}

The image is sub-divided into $4 \mathrm{x} 4$ pixels. The block is then converted into one dimensional vector of size $\mathrm{k}$ (where $\mathrm{k}=4 \mathrm{x} 4$ ) elements. Thus the input image is converted in to a training set of size $\mathrm{N}$ vectors, where $\mathrm{N}$ is computed using the equation (1).

$$
N=(m \times m) / k
$$

From each input block, the maximum pixel value Max and the minimum pixel value Min are calculated. If the Min and the Max values lie in the range 1 to 16, the input block belongs to the $1^{\text {st }}$ cluster. If the Min and Max values lie within the range 17 to 32 , the input block belongs to the $2^{\text {nd }}$ cluster. Similarly 16 such clusters are formed based on the minimum and maximum values. The ranges are (1-16), (17$32),(33-48) \ldots(225-240),(241-256)$. The average vectors of all the 16 clusters called the centroids are computed to form 16 code vectors of the codebook. Some of the input blocks that do not fall into any of the above clusters are used to generate the remaining (M-16) code vectors, where $M$ is the desired codebook size. Such blocks are categorized into high detail and low detail blocks. To categorize them as high detail and low detail blocks, the mean $(\bar{x})$ for each block is computed using the equation (2). The sum $\mathrm{S}$ of the difference between the mean and the individual pixel value is computed using the equation (3).

$$
\bar{x}=\frac{1}{m} \sum_{i=1}^{m} x_{i}
$$

$$
S=\sum_{i=1}^{m} a b s\left(x_{i}-\bar{x}\right)
$$

If $\mathrm{S}$ is greater than a threshold value, then the block is categorized into high detail block otherwise it is a low detail block. The threshold value depends up on the size $M$ of the codebook. Generally the edge blocks will be of high detail blocks. The edge blocks are given preference to preserve the edges in the image. This avoids ragged edges. Hence more number of edge blocks is selected when compared to the shade blocks while filling the remaining codebook. This improves the quality of the codebook.

\section{B. Categorization of blocks}

Total number of blocks for an image of size $256 \times 256$ pixels: 4096

\section{Lena image}

No. of blocks satisfying the MinMax condition: 956

No. of edge blocks: 1520

No. of shade blocks: 1620

\section{Cameraman image}

Min-Max Blocks: 1395

Edge Block: 1512

Shade Block: 1189

The codebook thus generated is optimized using the K-Means Clustering technique. The optimization of codebook improves the quality of the reconstructed image.

\section{Selection of Blocks for The Codebook}

The blocks satisfying the Minima condition are grouped into 16 clusters and the centroids of the clusters are taken thus forming first 16 codevectors. In case of a codebook of size 256, the remaining 240 codevectors are selected from the edge and shade blocks. Of these 240, 200 vectors are taken from edge blocks arbitrarily and the remaining 40 blocks are selected from the shade blocks. Thus a codebook of desired size is generated. Similarly for codebook of other sizes, the number of different types of blocks to be selected is given in TABLE I.

TABLE I: NO. OF Blocks OF DifFERENT Types SELECTED FOR VARIOUS CODEBOOK SizES.

\begin{tabular}{|c|c|c|c|}
\hline CB Size & Min-Max & Edge & Shade \\
\hline 64 & 16 & 40 & 8 \\
\hline 128 & 16 & 100 & 12 \\
\hline 256 & 16 & 200 & 40 \\
\hline 512 & 16 & 284 & 212 \\
\hline 1024 & 16 & 584 & 424 \\
\hline
\end{tabular}

The above numbers are fixed on trial basis based on the quality of the reconstructed images.

\section{K-Means Clustering}

The initial codebook thus created is optimized using the KMeans clustering, called Generalized Lloyd Algorithm. The training vectors are grouped into $\mathrm{M}$ clusters based on the equation (4). Pick up the codebook entry $\mathrm{y}_{\mathrm{i}}(\mathrm{k})$. Find all the training vectors that are closer to $\mathrm{y}_{\mathrm{i}}$ that satisfy

$$
d\left(x_{i,} y_{i}<d\left(x_{i} y_{j}\right) \text { for all } j \neq i\right.
$$

where the distance between the training vectors $x_{i}$ and the code vector $\mathrm{y}_{\mathrm{i}}$ is computed using the equation (5).

$$
d\left(x_{i}, y_{i}\right)=\sum_{i=1}^{k}\left|x_{i}-y_{i}\right|
$$

For each cluster, the centroid is computed. To compute the centroid, the Sum Vector $\operatorname{Sum}_{\mathrm{ij}}$ is computed using the equation (6).

$$
\operatorname{Sum}_{i j}=\sum_{i=1}^{c_{i}} x_{i j} \quad \text { where } j=1,2, \ldots, k
$$

where, $c_{i}$ is the cluster strength. Now every component of the Sum Vector is divided by the cluster strength $\mathrm{c}_{\mathrm{i}}$ to get the new centroid of the cluster.

$$
\text { Centroid }=\text { Sum }_{i j} / c_{i} \quad \text { where } i=1,2, \ldots . . M
$$

The codevector $y_{i}$ is replaced with the newly generated centroid to form the refined codebook. The above steps are repeated until the codebooks of the consecutive iterations converge.

\section{Algorithm}

Step1: The training vectors are grouped into M clusters based on the equations (4) and (5).

Step2: Compute the Sum Vector using the equation (6).

Step3: Compute the centroid of the each cluster using the equation (7).

Step4: Replace the existing codevectors with the new centroids to form the revised codebook. 
Step5: Repeat the steps 1 through 4 till the codebooks of consecutive iterations converge.

The PSNR value of the reconstructed image using the optimized codebook is high when compared to that of the image reconstructed using the initial codebook. The size of the codebook thus generated and optimized is reduced further using the MMSE method.

\section{E. Minimum Mean Square Error (MMSE) Method}

Minimum Mean Square Error (MMSE) was proposed by Lloyd [S. P. Lloyd, 1982] for Block Truncation Coding which is one other lossy image compression technique. In 4level MMSE technique, the Min and Max values are calculated for each block and a 4-level quantizer (a, b, c, d) is designed based on threshold value that is computed using the equation (8).

$$
t_{r}=\min +((\max -\min ) r / n)
$$

where $t_{r}$ represents the $r^{\text {th }}$ value of the threshold and $n$ is the number of quantization levels and four quantizing levels a, $\mathrm{b}, \mathrm{c}$ and $\mathrm{d}$ are calculated using the equation (9). Quantizer values are optimized.

$$
\left.\begin{array}{l}
a=\min \\
b=(2 \times \min +\max ) / 3 \\
c=(\max +2 \times \min ) / 3 \\
d=\max
\end{array}\right\}
$$

The compressed codevector is stored as a set of a bit plane and the two other statistical moments i.e. \{32-bit bit plane, a, $\mathrm{d}\}$. While reconstructing the image, $b$ and $c$ can be computed from the values $a$ and $d$. The entire codebook is thus a collection of sets of 2-bit bit planes and the respective statistical moments for all the codevectors. Each component of the codevector is compared against the threshold values $t r$, where $\mathrm{r}=1,2$ and 3 . If the component of the code vector is $<=\mathrm{t} 1$, then it is coded as 00 . If $>\mathrm{t} 1$ and $<=\mathrm{t} 2$, then it is coded as 01 . If the value is $>\mathrm{t} 2$ and $<=\mathrm{t} 3$, it is coded as 10 other wise 11 . Now the coded bitplane for any codevector will be like $[11,11,01,00,11,11,01,00,11,10,10,00,11$, $10,01,00]$ and the codevector is replaced with the set of coded bit plane, $a$ and $d$. Generally, a codeword takes $16 \times 8$ $=128$ bits. But after compression using 4-level MMSE, the codevector requires only $32+8+8=48$ bits. A compression rate of $62.5 \%$ is achieved.

\section{Compression achieved as a result of Normal VQ with a codebook of size 256 codewords}

Codebook Size $(256 \times 16 \times 8): 32768$ bits

Index Map Size $(64 \times 64 \times 8): 32768$ bits

Total Compressed Size $: 65536$ bits

Original image size $(256 \times 256$ pixels $): 65536$ pixels

Total number of Bits required by the original image (256 $\times 256 \times 8): 524288$ bits

Bit-rate $=$ Compressed Size $/$ Original Image Size = $65536 / 65536=1.00 \mathrm{bpp}$

Compression Rate $=100$-(Size of Compressed Image/Size of Original Image $\times 100)$

$$
\begin{aligned}
& =100-((65536 / 524288) * 100) \\
& =87.50 \%
\end{aligned}
$$

Compression achieved as a result of MMSE

Codebook Size $(256 \times 48) \quad: 12288$ bits
Index Map Size $(64 \times 64 \times 8: 32768$ bits

Total Compressed Size :45056 bits

Original image size $(256 \times 256): 65536$ pixels

Total Bits of the original image $(256 \times 256 \times 8): 524288$ bits

Bit-rate $=$ Total Compressed Size $/$ Original Image Size $=45056 / 65536=0.69 \mathrm{bpp}$

Compression Rate $=100$-(Size of Compressed Image/Size of Original Image $\times 100)$

$$
\begin{aligned}
& =100-((45056 / 524288) \times 100) \\
& =91.40 \%
\end{aligned}
$$

\section{MMSE Algorithm}

Step1: Get the initial codebook

Step2: For each codevector in the codebook, perform the following steps.

Step3: Calculate min and max values for each codevector

Step4: Calculate 4-level quantizer using equations (8) and (9)

Step5: Store Bitplane and quantizer levels

The codebook thus compressed using MMSE method is further compressed using the Interpolative method.

\section{F. Interpolative Method}

In this method, the bitplane generated using MMSE is further compressed by dropping the intermediate bit sequences as in Fig. 2. Hence the size of the bitplane is reduced from 32 bits to just 16 bits.

\begin{tabular}{|c|c|c|c|}
\hline $\mathrm{X} 1$ & $\mathbf{X 2}$ & $\mathrm{X} 3$ & $\mathrm{X} 4$ \\
\hline $\mathrm{X5}$ & $\mathrm{X} 6$ & $\mathrm{X} 7$ & $\mathrm{X} 8$ \\
\hline $\mathrm{X} 9$ & $\mathbf{X 1 0}$ & $\mathrm{X} 11$ & $\mathrm{X12}$ \\
\hline $\mathrm{X13}$ & $\mathrm{X} 14$ & $\mathrm{X15}$ & $\mathrm{X} 16$ \\
\hline
\end{tabular}

Fig 1. The pattern of dropping bits. The bold faced bits are dropped.

In decoding phase, the dropped bits are recovered by taking the arithmetic mean of the adjacent pixel values as given in equation (10).

$$
\left.\begin{array}{l}
x_{i}=\frac{1}{3}\left(x_{i-1}+x_{i+1}+x_{i+4}\right) \text { for } \mathrm{i}=2 \\
x_{i}=\frac{1}{2}\left(x_{i-1}+x_{i+4}\right) \text { for } \mathrm{i}=4 \\
x_{i}=\frac{1}{3}\left(x_{i-4}+x_{i+1}+x_{i+4}\right) \text { for } \mathrm{i}=5 \\
x_{i}=\frac{1}{4}\left(x_{i-4}+x_{i-1}+x_{i+1}+x_{i+4}\right) \text { fori }=7,10 \\
x_{i}=\frac{1}{3}\left(x_{i-4}+x_{i-1}+x_{i+4}\right) \text { for } \mathrm{i}=12 \\
x_{i}=\frac{1}{2}\left(x_{i-4}+x_{i+1}\right) \text { for } \mathrm{i}=13 \\
x_{i}=\frac{1}{3}\left(x_{i-1}+x_{i+1}+x_{i-4}\right) \text { for } \mathrm{i}=15
\end{array}\right\}
$$

As a result of this method, the codebook takes only 32 $(16+8+8)$ bits per vector. 


\section{Compression achieved as a result of Interpolative method}

Codebook Size $(256 \times 32): 8192$ bits

Index Map Size $(64 \times 64 \times 8): 32768$ bits

Total Compressed Size : 40960 bits

Original image size $(256 \times 256) \quad: 65536$ pixels

Total Bits of the original image $(256 \times 256 \times 8): 524288$ bits

Bit-rate $=$ Total Compressed Size / Original Image Size $=40960 / 65536=0.63 \mathrm{bpp}$

Compression Rate $=100$-(Size of Compressed

Image/Size of Original Image $\times 100)$

$$
\begin{aligned}
& =100-((40960 / 524288) \times 100) \\
& =92.19 \%
\end{aligned}
$$

Further compression is done to the Index Map using the Search Order Coding (SOC). The above two levels lead to lossy compression. But compression using SOC leads to lossless compression.

\section{G. Search Order Coding (SOC)}

In SOC, the feature of inter-block correlation is exploited. There are more chances for a processed block to be similar to the neighboring blocks. The index of the current block $\mathrm{cb}$ is compared with the index of the neighboring blocks in the given order $\mathrm{lb}, \mathrm{tb}, \mathrm{tlb}, \mathrm{lrb}$ as in the Fig. 2.

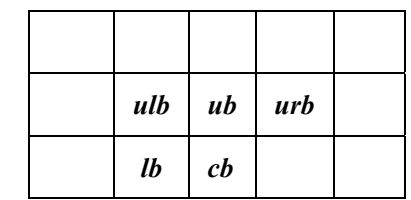

Fig. 2. Search Order of the Neighboring Indices

If the index of $\boldsymbol{c} \boldsymbol{b}$ is is equal to the index of $\boldsymbol{l} \boldsymbol{b}$, the index of the current block is coded as ' 00 '. If equal to the index of block $\boldsymbol{u b}$, the index is encoded as ' 01 '. If equal to the index of the block $\boldsymbol{u l b}$, the index is encoded as ' 10 '. If equal to the index of the block urb, the index is encoded as ' 11 '. If index is not equal to any of the neighboring blocks, then the original index of size 8 bits is stored ( 8 bits in the case of codebook of size 256). The total number of bits taken to store the encoded image is computed using the equation

$$
\mathrm{N}=\mathrm{N} 1+\mathrm{N} 2+\mathrm{N} 3+\mathrm{N} 4
$$

where N1-Number of blocks whose indices are equal to the index of the left block.

N2-Number of blocks whose indices are equal to the index of the upper block

N3-Number of blocks whose indices are equal to the index of the upper left block

N4-Number of blocks whose indices are equal to the index of the upper right block

The number of blocks that are not similar to the nearest neighbors is then computed equation (12) as

$$
n=(64 \times 64-N)
$$

With normal VQ, the bit-rate achieved with a codebook of size 256 is computed as

$$
\begin{array}{ll}
\text { Codebook Size }(256 \times 16 \times 8) & : 32768 \text { bits } \\
\text { Index Map Size }=64 \times 64 \times 8 & : 32768 \text { bits } \\
\text { Total No. of bits } & : 65536 \text { bits }
\end{array}
$$

Bit-rate $(65536 /(256 \times 256)) \quad: 1 \mathrm{bpp}$

With the proposed scheme, for the image Lena

Codebook Size $(256 \times 32)$

: 8192 bits

Index Map $=(2243 \times 8)+(1853 \times 2): 21650$ bits

Total No. of bits : 29842

Bit-rate $(29842 /(256 \times 256)) \quad: 0.46 \mathrm{bpp}$

Compression achieved as a result of Search Order Coding

For Lena image,

Codebook Size $(256 \times 32) \quad: 8192$

Index Map Size $((2243 \times 8)+(1853 \times 2)): 21650$

Total Compressed Size : 29842

Original image size $(256 \times 256): 65536$

Total Bits of the original image $(256 \times 256 \times 8): 524288$

Bit-rate $=$ Total Compressed Size / Original Image Size $=29842 / 65536=0.46 \mathrm{bpp}$

Compression Rate $=100$-(Size of Compressed Image/Size of Original Image $\times 100)$

$=100-((29842 / 524288) \times 100)$

$=94.31 \%$

\section{H. Decoding Stage}

In decoding stage, to reconstruct the compressed image, the codebook is decompressed in the reverse order in which the codebook is transformed into various forms through all the levels.

Step1: The reverse of Interpolation is done in which the dropped out bit sequences are reconstructed using the equations (10).

Step2: The reverse of MMSE is performed, in which the code vector is reconstructed from the 2-bit plane and its respective statistical moments.

Step3: The Index map is reconstructed based on the similarity of the neighboring blocks.

Step4: For each index in the index map, the corresponding codevector is coded to get the approximation of the input image.

\section{RESUlTS AND Discussion}

Experiments were carried out with standard images Cameraman, Boats, Bridge, Baboon, Lena and Kush of size $256 \times 256$ pixels. The input images taken for the study are given in Fig. 3.
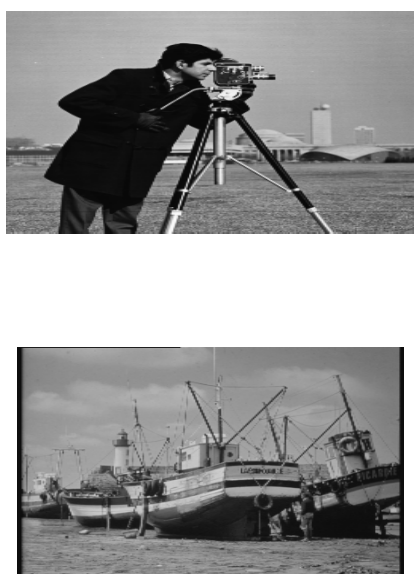


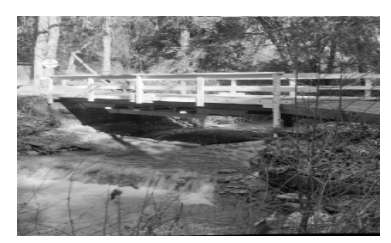

(a) Cameraman (b) Boats (c) Bridge
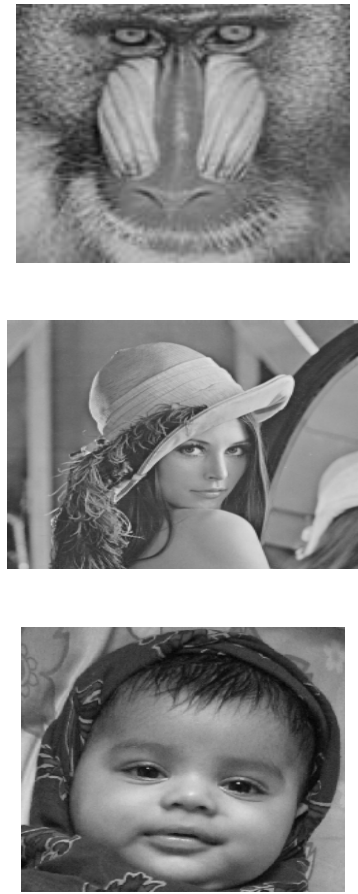

(d) Baboon

(e) Lena

(f) Kush

Fig. 3. Input images taken for the study

The PSNR value is taken as a measure of the quality of the reconstructed image and bpp is the measure of the compression efficiency. The algorithm is implemented using MATLAB 7.0 on Windows Operating System. The hardware used is the Intel ${ }^{\circledR} 3.06 \mathrm{GHz}$ Processor with 512 MB RAM. Table 1 gives the results obtained in terms of PSNR and bpp after every level of compression for standard images with codebooks of different sizes. For a codebook of size 64 , the bpp has been reduced from 0.50 to 0.26 . For a codebook of size 1024, the bpp has been reduced from 2.63 to 1.04 , which is a significant improvement.

In TABLE II, wit codebook of smaller sizes (64 and 128), the PSNR is not much affected, But the bpp has been reduced to half of the size that is obtained after VQEC. As the size of the codebook increases, there is a decrease in bpp as well as in PSNR.

In TABLE III, quality (PSNR) of the reconstructed images, the bpp and the Compression Rate (in \%) after every level of compression are given. A maximum of $96.21 \%$ is achieved with the proposed scheme, which is an appreciable value.

Linde, Buzo and Gray (LBG), Kekre's Proportionate Error Algorithm (KPE) [22], Simple Codebook Generation (SCG) and Ordered Codebook Generation (OCG) [23], Codebook Compression (CBC) [24], Training Set Partitioning (TSP) [25] methods are some of the existing codebook generation techniques. The results obtained with them are compared with that of the proposed scheme in
TABLE IV. With codebooks of lesser sizes, there is no much loss in PSNR, but the bpp has been reduced to a significant value.

In techniques such as LBG, KPE, SCG and OCG TSP, the PSNR obtained is little bit greater than that of the proposed method. But the bit-rate achieved in the proposed method is less when compared to that of all the methods. When compared to the TSP method, both the PSNR and the bpp values are better in the proposed method. With codebooks of smaller size, there is only little raise in the PSNR. But as the codebook size increases, the maximum difference in PSNR values between the existing methods and the proposed method is 3.32. But the proposed methods gives better compression rate when compared to all the existing methods mentioned in the study. In LBG method, with the codebook of size 1024, the bpp obtained is 2.63 . But the bpp achieved with the proposed method is only 1.04 which is a significant improvement. A minimum of 0.27 bpp is also achieved with the proposed method with the codebook of size 64 .

\section{CONCLUSION}

In this paper, we have presented a simple and efficient coding scheme for the compression of Image. The compression is done in four levels. In the first level, normal VQ is done, where a novel idea has been incorporated in generating the initial codebook with edge categorization. In the second level, the concept of MMSE is used to compress the codebook to contain the bit plane and the statistical moments of the codevectors. In the third level, using the concept of Interpolation, further compression is done to the codebook by reducing the size of the bit plane by dropping the bit sequences in the alternate positions. Finally the concept of Search Order Coding is performed to reduce the size of the Index Map. In each level, the compression rate is improved. At the end of the compression procedure, a significant amount of compression that is up to $94.31 \%$ is achieved. After normal VQ is performed, an average compression rate of $84.6 .5 \%$ is achieved. After every level of compression, the average compression rate is improved from $84.06 \%$ to $90.12 \%, 91.33 \%$ and $92.68 \%$ respectively. The simulation results show that the proposed scheme improves the compression efficiency by maintaining significant level of PSNR of the reconstructed image. The image crosses four levels of compression i. Normal VQ, ii. 4-level MMSE, iii. Interpolative Technique and iv. Search Order Coding. By incorporating the four levels of compression, it is noticed that a significant reduction in bit rate is achieved. The results of the proposed scheme, when compared to that of few existing methods, gives better results both in terms of bit-rate and the quality of the reconstructed images. With the higher codebook sizes, the existing methods give better PSNR values. But as far as the compression rate is concerned, only the proposed method gives a significant reduction in the bpp. This compression scheme can easily be extended to color images. This application is suitable for hand-held devices, where image processing applications play a vital role in present scenario. Since it is a lossy compression system, it is- suitable for Human Visual System (HVS), where loss in image data 
does not make much difference. In this paper, we have integrated the ideas incorporated in BTC and VQ to bring out the proposed scheme. Further enhancements to this kind of techniques and when integrated with other techniques, may lead to a better scheme when compared to JPEG standard in near future.

TABLE II: QUALITY OF THE RECONSTRUCTED IMAGES AND THE BPP OBTAINED AFTER EVERY LEVEL OF COMPRESSION FOR DiFFERENT IMAGES WITH DIFFERENT CODEBOOK SIZES

\begin{tabular}{|c|c|c|c|c|c|c|c|c|c|}
\hline \multirow{3}{*}{$\begin{array}{l}\text { CB } \\
\text { Size } \\
\end{array}$} & \multirow{3}{*}{ Images } & \multirow{2}{*}{\multicolumn{6}{|c|}{ IN Div }} & \multirow{2}{*}{\multicolumn{2}{|c|}{ SOC }} \\
\hline & & & & & & Interpo & ation & & \\
\hline & & PSNR & bpp & PSNR & bpp & PSNR & bpp & PSNR & bpp \\
\hline \multirow{8}{*}{64} & Cameraman & 28.43 & 0.50 & 28.16 & 0.42 & 27.46 & 0.41 & 27.46 & 0.23 \\
\hline & Baboon & 34.67 & 0.50 & 34.50 & 0.42 & 34.50 & 0.41 & 34.50 & 0.31 \\
\hline & Boats & 29.21 & 0.50 & 29.00 & 0.42 & 28.45 & 0.41 & 28.45 & 0.26 \\
\hline & Bridge & 26.94 & 0.50 & 26.81 & 0.42 & 26.58 & 0.41 & 26.58 & 0.31 \\
\hline & Lena & 31.47 & 0.50 & 31.22 & 0.42 & 31.02 & 0.41 & 31.02 & 0.25 \\
\hline & Kush & 31.86 & 0.50 & 31.67 & 0.42 & 31.46 & 0.41 & 31.46 & 0.27 \\
\hline & Goldhill & 34.27 & 0.50 & 34.12 & 0.42 & 33.92 & 0.41 & 33.92 & 0.26 \\
\hline & Average & 30.98 & 0.50 & 30.78 & 0.42 & 30.48 & 0.41 & 30.48 & 0.27 \\
\hline \multirow{8}{*}{128} & Cameraman & 29.66 & 0.69 & 29.29 & 0.53 & 28.31 & 0.50 & 28.31 & 0.30 \\
\hline & Baboon & 35.87 & 0.69 & 35.56 & 0.53 & 35.35 & 0.50 & 35.35 & 0.41 \\
\hline & Boats & 30.27 & 0.69 & 29.98 & 0.53 & 29.09 & 0.50 & 29.09 & 0.35 \\
\hline & Bridge & 27.75 & 0.69 & 27.54 & 0.53 & 27.24 & 0.50 & 27.24 & 0.41 \\
\hline & Lena & 32.71 & 0.69 & 32.37 & 0.53 & 31.80 & 0.50 & 31.80 & 0.33 \\
\hline & Kush & 32.99 & 0.69 & 32.73 & 0.53 & 32.31 & 0.50 & 32.31 & 0.36 \\
\hline & Goldhill & 35.81 & 0.69 & 35.57 & 0.53 & 35.21 & 0.50 & 35.21 & 0.36 \\
\hline & Average & 32.15 & 0.69 & 31.86 & 0.53 & 31.33 & 0.50 & 31.33 & 0.36 \\
\hline \multirow{8}{*}{256} & Cameraman & 30.92 & 1.00 & 30.41 & 0.69 & 28.21 & 0.63 & 28.21 & 0.44 \\
\hline & Baboon & 37.16 & 1.00 & 36.69 & 0.69 & 36.34 & 0.63 & 36.34 & 0.56 \\
\hline & Boats & 31.36 & 1.00 & 30.94 & 0.69 & 29.64 & 0.63 & 29.64 & 0.47 \\
\hline & Bridge & 28.60 & 1.00 & 28.32 & 0.69 & 27.66 & 0.63 & 27.66 & 0.54 \\
\hline & Lena & 33.97 & 1.00 & 33.42 & 0.69 & 32.62 & 0.63 & 32.62 & 0.46 \\
\hline & Kush & 34.29 & 1.00 & 33.81 & 0.69 & 33.15 & 0.63 & 33.15 & 0.49 \\
\hline & Goldhill & 37.60 & 1.00 & 37.06 & 0.69 & 36.55 & 0.63 & 36.55 & 0.49 \\
\hline & Average & 33.41 & 1.00 & 32.95 & 0.69 & 32.02 & 0.63 & 32.02 & 0.49 \\
\hline \multirow{8}{*}{512} & Cameraman & 32.13 & 1.56 & 31.44 & 0.94 & 29.26 & 0.81 & 29.26 & 0.66 \\
\hline & Baboon & 38.65 & 1.56 & 37.87 & 0.94 & 37.34 & 0.81 & 37.34 & 0.77 \\
\hline & Boats & 32.39 & 1.56 & 31.82 & 0.94 & 30.12 & 0.81 & 30.12 & 0.68 \\
\hline & Bridge & 29.60 & 1.56 & 29.19 & 0.94 & 28.20 & 0.81 & 28.20 & 0.75 \\
\hline & Lena & 35.37 & 1.56 & 34.57 & 0.94 & 33.16 & 0.81 & 33.16 & 0.68 \\
\hline & Kush & 35.53 & 1.56 & 34.92 & 0.94 & 33.97 & 0.81 & 33.97 & 0.71 \\
\hline & Goldhill & 39.32 & 1.56 & 38.39 & 0.94 & 37.55 & 0.81 & 37.55 & 0.70 \\
\hline & Average & 34.71 & 1.56 & 34.03 & 0.94 & 32.80 & 0.81 & 32.80 & 0.71 \\
\hline \multirow{8}{*}{1024} & Cameraman & 34.63 & 2.63 & 33.29 & 1.38 & 29.84 & 1.13 & 29.84 & 1.01 \\
\hline & Baboon & 40.60 & 2.63 & 39.31 & 1.38 & 38.42 & 1.13 & 38.42 & 1.09 \\
\hline & Boats & 34.42 & 2.63 & 33.38 & 1.38 & 30.79 & 1.13 & 30.79 & 1.00 \\
\hline & Bridge & 31.09 & 2.63 & 30.43 & 1.38 & 28.74 & 1.13 & 28.74 & 1.08 \\
\hline & Lena & 37.66 & 2.63 & 36.32 & 1.38 & 33.94 & 1.13 & 33.94 & 1.02 \\
\hline & Kush & 37.30 & 2.63 & 36.33 & 1.38 & 34.80 & 1.13 & 34.80 & 1.05 \\
\hline & Goldhill & 41.61 & 2.63 & 39.97 & 1.38 & 38.69 & 1.13 & 38.69 & 1.04 \\
\hline & Average & 36.76 & 2.63 & 35.56 & 1.38 & 33.60 & 1.13 & 33.60 & 1.04 \\
\hline
\end{tabular}

TABLE III: AVERAGE PSNR, BPP AND COMPRESSION RATE (\%) VALUES FOR DIFFERENT CODEBOOK SIZES

\begin{tabular}{|c|c|c|c|c|c|c|c|c|c|c|c|c|}
\hline \multirow{2}{*}{$\begin{array}{l}\text { CB } \\
\text { Size }\end{array}$} & \multicolumn{3}{|c|}{ CBEC } & \multicolumn{3}{|c|}{ MMSE } & \multicolumn{3}{|c|}{ Interpolative } & \multicolumn{3}{|c|}{ SOC } \\
\hline & PSNR & bpp & CR\% & PSNR & bpp & CR\% & PSNR & bpp & CR\% & PSNR & Bpp & CR\% \\
\hline 64 & 30.98 & 0.50 & 93.75 & 30.78 & 0.42 & 94.73 & 30.48 & 0.41 & 94.92 & 30.48 & 0.27 & 96.21 \\
\hline 128 & 32.15 & 0.69 & 91.41 & 31.86 & 0.53 & 93.36 & 31.33 & 0.50 & 93.75 & 31.33 & 0.36 & 95.47 \\
\hline 256 & 33.41 & 1.00 & 87.50 & 32.95 & 0.69 & 91.41 & 32.02 & 0.63 & 92.19 & 32.02 & 0.49 & 93.78 \\
\hline 512 & 34.71 & 1.56 & 80.47 & 34.03 & 0.94 & 88.28 & 32.80 & 0.81 & 89.84 & 32.80 & 0.71 & 91.06 \\
\hline 1024 & 36.76 & 2.63 & 67.19 & 35.56 & 1.38 & 82.81 & 33.60 & 1.13 & 85.94 & 33.60 & 1.04 & 86.88 \\
\hline \multicolumn{3}{|c|}{ Average } & 84.06 & & & 90.12 & & & 91.33 & & & 92.68 \\
\hline
\end{tabular}

* CR\% - Compression Rate in percentage

TABLE IV: PSNR AND BPP OBTAINED WITH THE EXISTING TECHNIQUES AND THE PROPOSED SCHEME.

\begin{tabular}{|c|c|c|c|c|c|c|c|c|c|c|c|c|c|c|}
\hline \multirow{2}{*}{$\begin{array}{l}\text { CB } \\
\text { Size }\end{array}$} & \multicolumn{2}{|c|}{ LBG } & \multicolumn{2}{|c|}{ KPE } & \multicolumn{2}{|c|}{ SCG } & \multicolumn{2}{|c|}{ OCG } & \multicolumn{2}{|c|}{ CBC } & \multicolumn{2}{|c|}{ TSP } & \multicolumn{2}{|c|}{$\mathbf{P M}$} \\
\hline & PSNR & bpp & PSNR & bpp & PSNR & bpp & PSNR & bpp & PSNR & bpp & PSNR & bpp & PSNR & Bpp \\
\hline 64 & 31.01 & 0.50 & 30.99 & 0.50 & 31.05 & 0.50 & 30.96 & 0.50 & 31.02 & 0.44 & 24.31 & 0.50 & 30.48 & 0.27 \\
\hline 128 & 32.15 & 0.69 & 32.14 & 0.69 & 31.87 & 0.69 & 32.11 & 0.69 & 31.98 & 0.56 & 25.82 & 0.69 & 31.33 & 0.36 \\
\hline 256 & 33.42 & 1.00 & 33.36 & 1.00 & 33.13 & 1.00 & 33.13 & 1.00 & 33.11 & 0.75 & 27.66 & 1.00 & 32.02 & 0.49 \\
\hline 512 & 34.90 & 1.56 & 34.87 & 1.56 & 34.44 & 1.56 & 34.44 & 1.56 & 34.40 & 1.06 & 28.84 & 1.56 & 32.80 & 0.71 \\
\hline 1024 & 36.92 & 2.63 & 36.87 & 2.63 & 36.17 & 2.63 & 36.26 & 2.63 & 35.98 & 1.63 & 29.97 & 2.63 & 33.60 & 1.04 \\
\hline
\end{tabular}




\section{REFERENCES}

[1] E. J. Delp and O. Robert, "Image Compression using Block Truncation Coding", IEEE Transactions on Communication, Col. COM-27, No. 9, 1335-1342, 1979.

[2] N. M. Nasrabadi and R. A. King, "A New Image Coding Technique using Transform Vector Quantization", Proceedings of International Conference on Acoustics, Speech and Signal Processing, San Diego, CA, pp. 29.9.1 - 29.9.4., 1984.

[3] J. S. Pan, Z. M. Lu, and S. H. Sun, "An Efficient Encoding Algorithm for Vector Quantization Based on Subvector Technique", IEEE Transactions on Image Processing, Vol 12, No. 3, March 2003.

[4] R. M.Gray, "Vector Quantization", IEEE Acoustics, Speech and Signal Processing Magazine, pp. 4-29, April 1984.

[5] Y. Linde, A. Buzo, and R. M. Gray, "An Algorithm for Vector Quantizer Design", IEEE Transactions on Communication", Vol. COM-28, No. 1, pp. 84-95, 1980

[6] J. Z. C. Lai, Y. C. Liaw, and J. Liu, " A Fast VQ Codebook Generation Algorithm using Codeword Displacement", Pattern Recognition, Vol. 41, No. 1, pp. 315-319, 2008.

[7] Y. C. Liaw, J. Z. C. Lai, and W. Lo, "Image Restoration of Compressed Image using Classified Vector Quantization", Pattern Recognition, Vol. 35, No. 2, pp. 181-192, 2002.

[8] N. M. Nasrabadi and Y. Feng, "Image Compression using Address Vector Quantization", IEEE Transactions on Communication, Vol. 38, No. 12, pp. 2166-2173, 1990.

[9] J. Foster, R. M. Gray, and M. O. Dunham, "Finite State Vector Quantization for Waveform Coding", IEEE Transactions on Information Theory, Vol. 31, No. 3, pp. 348-359, 1985.

[10] T. Kim, "Side Match and Overlap Match Vector Quantizers for Images", IEEE Transactions on Image Processing, Vol. 1, No. 2, pp. 170-185, 1992

[11] J. Z. C. Lai, Y. C. Liaw, W. Lo, "Artifact Reduction of JPEG Coded Images using Mean-Removed Classified Vector Quantization", Signal Processing, Vol. 82, No. 10, pp. 1375-1388, 2002.

[12] C. C. I. Katsavounidis, J. Kuo, and Z. Zhang, "A New Initialization Technique for Generalized Lloyd Iteration”, IEEE Signal Processing Letters, Vol. 1, No. 10, October 1994.

[13] Gersho and R. M. Gray, "Vector Quantization and Signal Compression" Dordrecht, The Netherlands: Kluwer, 1992.

[14] K. Somasundaram and S. Vimala, "Fast Codebook Generation for Quantization using Ordered Pairwise Nearest Neighbor with
Multiple Merging", In Proceedings of the IEEE International Conference on Emerging Trends in Electrical and Computer Technology (ICETECT), pp. 581-588, 2011.

[15] X. Wu and K. Zhang, "A Better Tree Structured Vector Quantizer", IEEE Proceedings of the Data Compression Conference, Snowbrid, UT, pp. 392-401, 1991.

[16] P. Franti, T. Kaukoranta and O. Nevalainen, "On the Splitting Method for VQ Codebook Generation", optical Engineering, Vol. 36, pp. 3043-3051, Nov' 1997.

[17] W. Equitz, "A New Vector Quantization Clustering Algorithm", IEEE Transactions Acoustics, Speech and Signal Processing, Vol. 37, No. 10, October 1989

[18] A. Gersho and V. Cuperman, "Vector Quantization: A patternmatching technique for speech coding," IEEE Communications Magazine, pp.15-21, 1983.

[19] K. Somasundram, I. Kaspar Raj, “An Image compression Scheme based on Predictive and Interpolative Absolute Moment Block Truncation Coding", GVIP Journal, Volume 6, Issue 4, December 2006

[20] P. Franti, O. Nevalainen, and T. Kaukoranta,"Compression of Digital Images by Block Truncation Coding:A Survey", The Computer Journal, Vol.37, No.4, 1994.

[21] S. P. Lloyd, 1982: "Least Square Quantization in PCM", IEEE Transactions on Information and Theory, Vol. 28, No. 2, pp. 129-137.

[22] H. B. Kekre and T. K. Sarode, "Fast Codebook Search Algorithm for Vector Quantization using Sorting Technique", Proceedings of International Conference on Advances in Computing, Communication and Control (ICAC3), 2009.

[23] K. Somasundaram and S. Vimala, "Simple and Fast Ordered Codebook for Vector Quantization", Proceedings of the National Conference on Image Processing (NCIMP 2010) held on Gandhigram Rural University, India, Allied Publishers Pvt. Ltd., pp. 3-7, 2010

[24] S. Vimala, "Low Bit-Rate Vector Quantization with Codebook Compression and Index Compression", Proceedings of the International Conference on Computing: Upgrades and Trends (CUT 2010), APH Publishing Corporation, New Delhi, Chapter-22, pp. 285-298, 2010.

[25] S. Vimala, "Techniques for Generating Initial Codebook for Vector quantization", Proceedings of the International Conference on Electronics Computer Technology (ICECT 2011), IEEE Catalog No.: CFP1195F-ART, No. 4, pp. 201-208, 2011. 